\title{
THERMAL INSTABILITY OF A COUPLE-STRESS FERROMAGNETIC FLUID IN THE PRESENCE OF VARIABLE GRAVITY FIELD AND HORIZONTAL MAGNETIC FIELD WITH HALL CURRENTS SATURATING IN A POROUS MEDIUM
}

\author{
D.O.I - 10.51201/Jusst12558 \\ http://doi.org/10.51201/Jusst12558 \\ Sudhir Kumar Pundir, Pulkit Kumar Nadian*, Rimple Pundir \\ Department of Mathematics \\ S.D. (P.G.) College, Muzaffarnagar, U.P.-251001, India
}

\begin{abstract}
The thermal instability of a couple-stress ferromagnetic fluid in the presence of variable gravity field and horizontal magnetic field with hall currents is considered, which saturates in a porous medium. We have used a linear stability theory and normal mode technique to discover the precise solution for a layer of couple-stress ferromagnetic fluid contained between two free boundaries. A dispersion relation governing the effect of medium permeability, couple-stress, magnetic field and hall current is derived. For the case of stationary convection, we have found that these parameters have both stabilizing and destabilizing effect under certain conditions. From the present study, we have got additionally determined that magnetization has a stabilizing effect on the system. Graphs in each case have been plotted by giving numerical values to these parameters. In the absence of magnetic field (hence hall current), the principle of exchange of stabilities is found to hold true under certain conditions.
\end{abstract}

Keywords: Thermal instability, couple-stress fluid, ferromagnetic fluid, magnetic field, hall current.

Mathematical Subject Classification 2020: 76A05, 76A10, 76D05, 76E06, 76E25, 76S05, 76W05.

\section{Introduction}

The developing significance of using non-Newtonian fluids in modern technology and industries has led diverse researchers to strive numerous flow problems associated with several nonNewtonian fluids. One such fluid that has attracted the attention of research workers during the last four decades is the couple-stress fluid. The theory of couple-stress fluids initiated by Stokes $[1,2]$ is a generalization of the classical theory of viscous fluids, which allows for the presence of couple-stresses and body couples in the fluid medium. Sunil et al. [3] have mentioned the effect of Hall currents on thermosolutal instability of compressible Rivlin-Ericksen fluid. Rani 
and Tomar $[4,5]$ have investigated thermal and thermosolutal convection problem of micropolar fluid subjected to Hall current. Kumar [6] have examined the effect of Hall currents on thermal instability of compressible dusty viscoelastic fluid saturated in a porous medium subjected to vertical magnetic field.

There are diverse stability problems on ferromagnetic fluids. The convective instability, additionally referred to as Bénard convection (Chandrasekhar [7]) is one of the instability of ferromagnetic fluid. Rosensweig [8] has given a definite introduction about magnetic liquids in his monograph. Finlayson [9] have studied the convective instability of ferromagnetic fluid for a fluid layer heated from below in the presence of uniform vertical magnetic field. The effect of Hall current on thermal instability has additionally been mentioned by many authors ( Raghavachar et al. [10], Sharma et al. [11], Gupta et al. [12], [13]). Aggarwal and Makhija [14] have studied the effect of Hall current on thermal stability of ferromagnetic fluids heated from below in porous medium in the presence of horizontal magnetic field.

In the present study, we have discussed the effect of Hall current on thermal instability of couple-stress ferromagnetic fluid in the presence of variable gravity field and horizontal magnetic field saturating in a porous medium. Recently, some stability problems about couplestress ferromagnetic fluid have been discussed by Nadian et al. [15, 16, 17, 18]. In the present problem, we have assumed that the gravity is varying as $g=\lambda g_{0}$ where $g_{0}$ is the value of $g$ at the surface of the Earth, which is always positive and $\lambda$ can be positive or negative.

\section{Mathematical Formulation of the Problem}

We consider an infinite, incompressible, electrically non-conducting and thin layer of couplestress ferromagnetic fluid which is bounded by the planes $z=0$ and $z=d$ as shown in Fig. 1. The fluid layer is heated from below so that a uniform temperature gradient $\beta=\left|\frac{d T}{d z}\right|$ is maintained within the fluid. The whole system is acted upon by a uniform vertical magnetic field $\boldsymbol{H}(0,0, H)$ and variable gravity field $g(0,0,-g)$, where $g=\lambda g_{0}$. Furthermore, the couple-stress ferromagnetic fluid layer is assumed to be flowing through an isotropic and homogeneous porous medium of porosity $\varepsilon$.

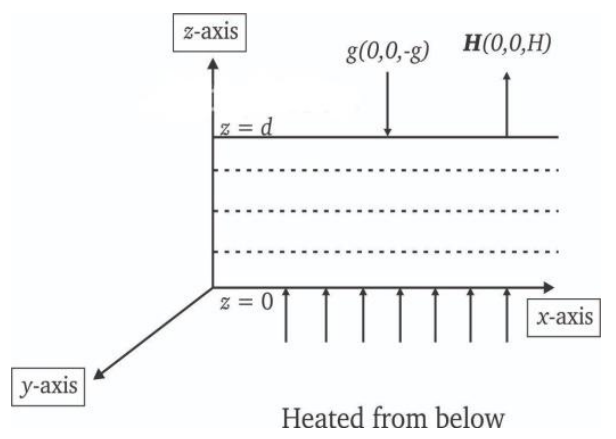

Fig. 1 Geometrical Configuration 
The equation of conservation of momentum, continuity, temperature and equation of density for the above model are as follows:

$$
\begin{gathered}
\frac{1}{\varepsilon}\left[\frac{\partial \boldsymbol{q}}{\partial t}+\frac{1}{\varepsilon}(\boldsymbol{q} \cdot \nabla) \boldsymbol{q}\right]=-\frac{1}{\rho_{0}} \nabla p+\boldsymbol{g}\left(1+\frac{\delta \rho}{\rho_{0}}\right)-\frac{1}{k_{1}} v \boldsymbol{q}+\frac{1}{\rho_{0}} \boldsymbol{M} \cdot \nabla \boldsymbol{H}+\left(v-\frac{\mu^{\prime}}{\rho_{0}} \nabla^{2}\right) \nabla^{2} \boldsymbol{q}+\frac{\mu_{e}}{4 \pi \rho_{0}}[(\nabla \times \boldsymbol{H}) \times \boldsymbol{H}], \\
\nabla \cdot \boldsymbol{q}=0, \\
E \frac{\partial T}{\partial t}+(\boldsymbol{q} \cdot \nabla) T=\kappa_{T} \nabla^{2} T, \\
\rho=\rho_{0}\left[1-\alpha\left(T-T_{0}\right)\right],
\end{gathered}
$$

where, $\boldsymbol{q}\left(u_{1}, u_{2}, u_{3}\right)$ is the fluid velocity, $p$ is the fluid pressure, $\rho$ is the fluid density, $\rho_{0}$ is the reference density, $T$ is the temperature, $T_{0}$ is the reference temperature, $\alpha$ is the thermal coefficient of expansion, $\mu_{e}$ is the magnetic permeability, $\mu$ is the couple-stress viscosity, $v$ is the kinematic viscosity, $\kappa_{T}$ is the thermal diffusivity, $E=\varepsilon+(1-\varepsilon) \frac{\rho_{s} c_{s}}{\rho_{0} c_{v}}$ (where, $\rho_{s}, c_{s}, c_{v}$ denote the density of the solid (porous) material, heat capacity of the solid (porous) material and heat capacity of the fluid at constant volume), $\boldsymbol{M}$ is the magnetization, $\nabla \boldsymbol{H}$ is the magnetic field gradient.

In presence of Hall current, the Maxwell's equations are given by,

$$
\varepsilon \frac{\partial \boldsymbol{H}}{\partial t}=\nabla \times(\boldsymbol{q} \times \boldsymbol{H})+\varepsilon \eta \nabla^{2} \boldsymbol{H}-\frac{\varepsilon}{4 \pi N e} \nabla \times[(\nabla \times \boldsymbol{H}) \times \boldsymbol{H}]
$$

and

$$
\nabla . \boldsymbol{H}=0,
$$

where, $\boldsymbol{H}$ is the magnetic field intensity.

Now, we consider that the magnetization is independent of magnetic field intensity but depend upon the temperature so that $M=M(T)$. As a first approximation, we consider that

$$
M=M_{0}\left[1-\gamma\left(T-T_{0}\right)\right]
$$

where, $\gamma=\frac{1}{M_{0}}\left(\frac{\partial M}{\partial T}\right)_{H}$ and $M_{0}$ is the magnetization at $T=T_{0}$ with $T_{0}$ being the reference temperature.

\section{Basic State and Perturbation Equations}

The basic state of which we wish to examine the stability is characterized by,

$\boldsymbol{q}=(0,0,0), p=p(z), \rho=\rho(z)=\rho_{0}(1+\alpha \beta z), T=T_{0}-\beta z, \boldsymbol{H}=(0,0, H), M=M_{0}(1+\gamma \beta z), \boldsymbol{M}=M(z)$

Here, $\beta$ may be either positive or negative. 
Assuming small perturbations around the basic state and let $\boldsymbol{q}\left(u_{1}, u_{2}, u_{3}\right), \boldsymbol{h}\left(h_{x}, h_{y}, h_{z}\right), \theta, \delta \rho, \delta p$ and $\delta M$ denote respectively the perturbations in fluid velocity, magnetic field, temperature, density, pressure and magnetization. Hence, the perturbed flow may be represented as,

$\boldsymbol{q}=(0,0,0)+\left(u_{1}, u_{2}, u_{3}\right), \boldsymbol{h}=(0,0, H)+\left(h_{x}, h_{y}, h_{z}\right), T=T(z)+\theta, \rho=\rho(z)+\delta \rho, p=p(z)+\delta p, M=M(z)+\delta M$.

Linearizing the equations in perturbation and reading the perturbation into normal modes, we anticipate that the perturbation quantity are of the form,

$$
\left(u_{3}, \theta, \zeta, \xi, h_{z}\right)=[W(z), \Theta(z), Z(z), X(z), K(z)] \cdot e^{\left\{i\left(k_{x} x+k_{y} y\right)+n t\right\}},
$$

where, $k_{x}$ and $k_{y}$ are wave numbers in $x$ and $y$ directions respectively and $k=\left(k_{x}^{2}+k_{y}^{2}\right)^{1 / 2}$ is the resultant wave number of the disturbance and $n$ is the frequency of any arbitrary disturbance (that is generally a complex constant).

We eliminate the physical quantities using the non-dimensional parameters $a=k d, \sigma=\frac{n d^{2}}{v}, p_{1}=\frac{v}{\kappa_{T}}, p_{2}=\frac{v}{\eta}$,

$F=\frac{\mu^{\prime}}{\rho_{0} d^{2} v}, P_{1}=\frac{k_{1}}{d^{2}}, D^{*}=d D$. Also dropping $(*)$ for convenience, we obtain,

$\left(D^{2}-a^{2}\right)\left[\left(\frac{\sigma}{\varepsilon}+\frac{1}{P_{1}}\right)+F\left(D^{2}-a^{2}\right)^{2}-\left(D^{2}-a^{2}\right)\right] W+\frac{\lambda \alpha a^{2} d^{2}}{v}\left(g_{0}-\frac{\gamma M_{0} \nabla H}{\rho_{0} \alpha \lambda}\right) \Theta-\frac{\mu_{e} H d}{4 \pi \rho_{0} v}\left(D^{2}-a^{2}\right) D K=0$,

$$
\left[\left(\frac{\sigma}{\varepsilon}+\frac{1}{P_{1}}\right)+F\left(D^{2}-a^{2}\right)^{2}-\left(D^{2}-a^{2}\right)\right] Z=\frac{\mu_{e} H d}{4 \pi \rho_{0} v} D X,
$$

$$
\left(D^{2}-a^{2}-\sigma p_{2}\right) X=-\frac{H d}{\varepsilon \eta} D Z-\frac{H}{4 \pi N e \eta d}\left(D^{2}-a^{2}\right) D K
$$

$$
\left(D^{2}-a^{2}-\sigma p_{2}\right) K=-\frac{H d}{\varepsilon \eta} D W+\frac{H d}{4 \pi N e \eta} D X,
$$

$$
\left(D^{2}-a^{2}-E \sigma p_{1}\right) \Theta=-\frac{\beta d^{2}}{\kappa_{T}} \mathrm{~W} .
$$

Now, eliminating $X, \Theta, K, Z$ among Eqs. (11) - (15), we obtain the stability governing equation,

$$
\begin{aligned}
& \lambda R_{f} a^{2} W=\left(D^{2}-a^{2}\right)\left\{\left(\frac{\sigma}{\varepsilon}+\frac{1}{P}\right)+F\left(D^{2}-a^{2}\right)^{2}-\left(D^{2}-a^{2}\right)\right\}\left(D^{2}-a^{2}-E \sigma p_{1}\right) W \\
& +\frac{\frac{Q}{\varepsilon} D^{2}\left(D^{2}-a^{2}\right)\left[\frac{Q}{\varepsilon} D^{2}+\left(D^{2}-a^{2}-\sigma p_{2}\right)\left\{\left(\frac{\sigma}{\varepsilon}+\frac{1}{P_{1}}\right)+F\left(D^{2}-a^{2}\right)^{2}-\left(D^{2}-a^{2}\right)\right\}\right]\left(D^{2}-a^{2}-E \sigma p_{1}\right) W}{\left(D^{2}-a^{2}-\sigma p_{2}\right)\left[\frac{Q}{\varepsilon} D^{2}+\left(D^{2}-a^{2}-\sigma p_{2}\right)\left\{\left(\frac{\sigma}{\varepsilon}+\frac{1}{P_{1}}\right)+F\left(D^{2}-a^{2}\right)^{2}-\left(D^{2}-a^{2}\right)\right\}\right]+M_{h} D^{2}\left(D^{2}-a^{2}\right)\left\{\left(\frac{\sigma}{\varepsilon}+\frac{1}{P_{1}}\right)+F\left(D^{2}-a^{2}\right)^{2}-\left(D^{2}-a^{2}\right)\right\}}
\end{aligned}
$$


where, $R_{f}=\frac{\alpha \beta d^{4}}{v \kappa_{T}}\left(g_{0}-\frac{\gamma M_{0} \nabla H}{\rho_{0} \alpha \lambda}\right)$ is the Rayleigh number for ferromagnetic fluid, $Q=\frac{\mu_{e} H^{2} d^{2}}{4 \pi \rho_{0} v \eta}$ is the Chandrasekhar number and $M_{h}=\left(\frac{H}{4 \pi N e \eta}\right)^{2}$ is the Hall current parameter.

Now, the appropriate boundary condition (when we take the case of two free boundaries) are, $W=0, D^{2} W=0, D^{4} W=0, \Theta=0, Z=0, X=0, D Z=0, D X=0, D K=0$ at $z=0$ and $z=1$.

Here, it is obvious that all even order derivatives of $W$ at the boundaries vanish. Therefore, the solution of Eq. (16) characterizing the lowest mode is,

$$
W=W_{0} \sin \pi z, \text { where } W_{0} \text { is a constant. }
$$

Now, using Eq. (18) in Eq. (16), we get,

$$
\begin{aligned}
& R_{1}=\frac{(1+x)}{\lambda x}\left\{\left(\frac{i \sigma_{1}}{\varepsilon}+\frac{1}{P}\right)+F_{1}(1+x)^{2}+(1+x)\right\}\left(1+x+E i \sigma_{1} p_{1}\right) \\
& +\frac{Q_{1}}{\lambda x \varepsilon} \frac{(1+x)\left[\frac{Q_{1}}{\varepsilon}+\left(1+x+i \sigma_{1} p_{2}\right)\left\{\left(\frac{i \sigma_{1}}{\varepsilon}+\frac{1}{P}\right)+F_{1}(1+x)^{2}+(1+x)\right\}\right]\left(1+x+E i \sigma_{1} p_{1}\right)}{\left(1+x+i \sigma_{1} p_{2}\right)\left[\frac{Q_{1}}{\varepsilon}+\left(1+x+i \sigma_{1} p_{2}\right)\left\{\left(\frac{i \sigma_{1}}{\varepsilon}+\frac{1}{P}\right)+F_{1}(1+x)^{2}+(1+x)\right\}\right]+M_{h}(1+x)\left\{\left(\frac{i \sigma_{1}}{\varepsilon}+\frac{1}{P}\right)+F_{1}(1+x)^{2}+(1+x)\right\}}
\end{aligned}
$$

where, $x=\frac{a^{2}}{\pi^{2}}, i \sigma_{1}=\frac{\sigma}{\pi^{2}}, F_{1}=\pi^{2} F, R_{1}=\frac{R_{f}}{\pi^{4}}, Q_{1}=\frac{Q}{\pi^{2}}, P=\pi^{2} P_{1}$.

\section{Analytical Discussion}

\section{Stationary Convection}

When stability sets in at stationary convection, the marginal state will be characterized by $\sigma_{1}=0$. So, put $\sigma_{1}=0$ in Eq. (19), we get,

$$
R_{1}=\frac{(1+x)^{2}}{\lambda x}\left\{\frac{1}{P}+F_{1}(1+x)^{2}+(1+x)\right\}+\frac{Q_{1}}{\lambda x \varepsilon} \frac{(1+x)\left\{\frac{Q_{1}}{\varepsilon}+\frac{(1+x)}{P}+F_{1}(1+x)^{3}+(1+x)^{2}\right\}}{\left\{\frac{Q_{1}}{\varepsilon}+\frac{(1+x)}{P}+F_{1}(1+x)^{3}+(1+x)^{2}\right\}+M_{h}\left\{\frac{(1+x)}{P}+F_{1}(1+x)^{3}+(1+x)^{2}\right\}} .
$$

This relation expresses the modified Rayleigh number $R_{1}$ as a function of the parameters $P, F_{1}, Q_{1}, M_{h}$ and dimensionless wave number $x$. Now, to have a look at the effect of medium permeability parameter, couple-stress parameter, magnetic field parameter and Hall current parameter, we examine the behavior of $\frac{d R_{1}}{d P}, \frac{d R_{1}}{d F_{1}}, \frac{d R_{1}}{d Q_{1}}$ and $\frac{d R_{1}}{d M_{h}}$.

So, by Eq. (20), we have, 


$$
\frac{d R_{1}}{d P}=\frac{(1+x)^{2}}{\lambda x P^{2}}\left[\frac{M_{h} Q_{1}^{2}}{\varepsilon^{2}\left[\frac{Q_{1}}{\varepsilon}+\left(1+M_{h}\right)\left\{\frac{(1+x)}{P}+F_{1}(1+x)^{3}+(1+x)^{2}\right\}\right]^{2}}-1\right],
$$

which indicates that medium permeability has a stabilizing effect on the system under the condition,

$$
\lambda>0, M_{h} Q_{1}^{2}>\varepsilon^{2}\left[\frac{Q_{1}}{\varepsilon}+\left(1+M_{h}\right)\left\{\frac{(1+x)}{P}+F_{1}(1+x)^{3}+(1+x)^{2}\right\}\right]^{2}
$$

and $\lambda<0, M_{h} Q_{1}^{2}<\varepsilon^{2}\left[\frac{Q_{1}}{\varepsilon}+\left(1+M_{h}\right)\left\{\frac{(1+x)}{P}+F_{1}(1+x)^{3}+(1+x)^{2}\right\}\right]^{2}$.

Also, medium permeability has a destabilizing effect on the system under the condition,

$$
\begin{aligned}
& \lambda>0, M_{h} Q_{1}^{2}<\varepsilon^{2}\left[\frac{Q_{1}}{\varepsilon}+\left(1+M_{h}\right)\left\{\frac{(1+x)}{P}+F_{1}(1+x)^{3}+(1+x)^{2}\right\}\right]^{2} \\
& \text { and } \lambda<0, M_{h} Q_{1}^{2}>\varepsilon^{2}\left[\frac{Q_{1}}{\varepsilon}+\left(1+M_{h}\right)\left\{\frac{(1+x)}{P}+F_{1}(1+x)^{3}+(1+x)^{2}\right\}\right]^{2} .
\end{aligned}
$$

In the absence of magnetic field, Eq. (21) becomes,

$$
\frac{d R_{1}}{d P}=-\frac{(1+x)^{2}}{\lambda x P^{2}}
$$

which indicates that medium permeability has a stabilizing effect for $\lambda<0$ and destabilizing effect for $\lambda>0$.

$$
\frac{d R_{1}}{d F_{1}}=\frac{(1+x)^{4}}{\lambda x}\left[1-\frac{M_{h} Q_{1}^{2}}{\varepsilon^{2}\left[\frac{Q_{1}}{\varepsilon}+\left(1+M_{h}\right)\left\{\frac{(1+x)}{P}+F_{1}(1+x)^{3}+(1+x)^{2}\right\}\right]^{2}}\right],
$$

which indicates that couple-stress has a stabilizing effect on the system under the condition,

$$
\lambda>0, M_{h} Q_{1}^{2}<\varepsilon^{2}\left[\frac{Q_{1}}{\varepsilon}+\left(1+M_{h}\right)\left\{\frac{(1+x)}{P}+F_{1}(1+x)^{3}+(1+x)^{2}\right\}\right]^{2}
$$

and $\lambda<0, M_{h} Q_{1}^{2}>\varepsilon^{2}\left[\frac{Q_{1}}{\varepsilon}+\left(1+M_{h}\right)\left\{\frac{(1+x)}{P}+F_{1}(1+x)^{3}+(1+x)^{2}\right\}\right]^{2}$.

Also, couple-stress has a destabilizing effect on the system under the condition,

$$
\lambda>0, M_{h} Q_{1}^{2}>\varepsilon^{2}\left[\frac{Q_{1}}{\varepsilon}+\left(1+M_{h}\right)\left\{\frac{(1+x)}{P}+F_{1}(1+x)^{3}+(1+x)^{2}\right\}\right]^{2}
$$


and $\lambda<0, M_{h} Q_{1}^{2}<\varepsilon^{2}\left[\frac{Q_{1}}{\varepsilon}+\left(1+M_{h}\right)\left\{\frac{(1+x)}{P}+F_{1}(1+x)^{3}+(1+x)^{2}\right\}\right]^{2}$.

In the absence of magnetic field or hall current, Eq. (22) becomes,

$$
\frac{d R_{1}}{d F_{1}}=\frac{(1+x)^{4}}{\lambda x},
$$

which clearly indicates that couple-stress has a stabilizing effect for $\lambda>0$ and destabilizing effect for $\lambda<0$.

$$
\frac{d R_{1}}{d Q_{1}}=\frac{(1+x)}{\lambda \varepsilon x}\left[\frac{\frac{M_{h} Q_{1}}{\varepsilon}\left\{\frac{(1+x)}{P}+F_{1}(1+x)^{3}+(1+x)^{2}\right\}+\left\{\frac{Q_{1}}{\varepsilon}+\frac{(1+x)}{P}+F_{1}(1+x)^{3}+(1+x)^{2}\right\} \cdot\left[\frac{Q_{1}}{\varepsilon}+\left(1+M_{h}\right)\left\{\frac{(1+x)}{P}+F_{1}(1+x)^{3}+(1+x)^{2}\right\}\right]}{\left[\frac{Q_{1}}{\varepsilon}+\left(1+M_{h}\right)\left\{\frac{(1+x)}{P}+F_{1}(1+x)^{3}+(1+x)^{2}\right\}\right]^{2}}\right],
$$

which clearly indicates that magnetic field has a stabilizing effect on the system for $\lambda>0$ and destabilizing effect for $\lambda<0$.

$$
\frac{d R_{1}}{d M_{h}}=-\frac{Q_{1}(1+x)}{\lambda \varepsilon x}\left\{\frac{Q_{1}}{\varepsilon}+\frac{(1+x)}{P}+F_{1}(1+x)^{3}+(1+x)^{2}\right\} \cdot \frac{\left\{\frac{(1+x)}{P}+F_{1}(1+x)^{3}+(1+x)^{2}\right\}}{\left[\frac{Q_{1}}{\varepsilon}+\left(1+M_{h}\right)\left\{\frac{(1+x)}{P}+F_{1}(1+x)^{3}+(1+x)^{2}\right\}\right]^{2}},
$$

which clearly indicates that Hall current has a stabilizing effect on the system for $\lambda<0$ and destabilizing effect for $\lambda>0$.

To see the effect of magnetization, we examine the effect of $\frac{d R}{d M_{0}}$ analytically.

$$
\begin{array}{r}
\frac{d R}{d M_{0}}=\left[\frac{\pi^{4}(1+x)^{2}}{\lambda x}\left\{\frac{1}{P}+F_{1}(1+x)^{2}+(1+x)\right\}+\frac{Q_{1} \pi^{4}}{\lambda x \varepsilon} \frac{(1+x)\left\{\frac{Q_{1}}{\varepsilon}+\frac{(1+x)}{P}+F_{1}(1+x)^{3}+(1+x)^{2}\right\}}{\left\{\frac{Q_{1}}{\varepsilon}+\frac{(1+x)}{P}+F_{1}(1+x)^{3}+(1+x)^{2}\right\}+M_{h}\left\{\frac{(1+x)}{P}+F_{1}(1+x)^{3}+(1+x)^{2}\right\}}\right] . \\
\left(1-\frac{\gamma M_{0} \nabla H}{\rho_{0} \alpha g_{0} \lambda}\right)^{-2} \cdot\left(\frac{\gamma \nabla H}{\rho_{0} \alpha g_{0} \lambda}\right),
\end{array}
$$

which clearly indicates that magnetization has a stabilizing effect on the system for both $\lambda>0$ and $\lambda<0$.

\section{The case of oscillatory modes}

Multiplying Eq. (11) by the conjugate of $W$ i.e. $W^{*}$ and integrate over the range of $z$ and making use of Eqs. (12) - (15) together with boundary conditions (17), we get,

$$
\begin{gathered}
\left(\frac{\sigma}{\varepsilon}+\frac{1}{P_{1}}\right) I_{1}+I_{2}+F I_{3}+d^{2}\left\{\left(\frac{\sigma^{*}}{\varepsilon}+\frac{1}{P_{1}}\right) I_{4}+I_{5}+F I_{6}+\frac{\mu_{e} \varepsilon \eta}{4 \pi \rho_{0} v}\left(I_{7}+p_{2} \sigma I_{8}\right)\right\}+\frac{\mu_{e} \varepsilon \eta}{4 \pi \rho_{0} v}\left(I_{9}+p_{2} \sigma^{*} I_{10}\right) \\
-\frac{\lambda \alpha a^{2} \kappa_{T}}{\beta v}\left(g_{0}-\frac{\gamma M_{0} \nabla H}{\rho_{0} \alpha \lambda}\right)\left(I_{11}+E p_{1} \sigma^{*} I_{12}\right)=0,
\end{gathered}
$$

where,

$$
I_{1}=\int\left(|D W|^{2}+a^{2}|W|^{2}\right) d z, I_{2}=\int\left(\left|D^{2} W\right|^{2}+2 a^{2}|D W|^{2}+a^{4}|W|^{2}\right) d z
$$




$$
\begin{gathered}
I_{3}=\int\left(\left|D^{3} W\right|^{2}+3 a^{2}\left|D^{2} W\right|^{2}+3 a^{4}|D W|^{2}+a^{6}|W|^{2}\right) d z, I_{4}=\int\left(|Z|^{2}\right) d z, \\
I_{5}=\int\left(|D Z|^{2}+a^{2}|Z|^{2}\right) d z, I_{6}=\int\left(\left|D^{2} Z\right|^{2}+2 a^{2}|D Z|^{2}+a^{4}|Z|^{2}\right) d z, \\
I_{7}=\int\left(|D X|^{2}+a^{2}|X|^{2}\right) d z, I_{8}=\int|X|^{2} d z, \\
I_{9}=\int\left(\left|D^{2} K\right|^{2}+2 a^{2}|D K|^{2}+a^{4}|K|^{2}\right) d z, I_{10}=\int\left(|D K|^{2}+a^{2}|K|^{2}\right) d z, \\
I_{11}=\int\left(|D \Theta|^{2}+a^{2}|\Theta|^{2}\right) d z, I_{12}=\int\left(|\Theta|^{2}\right) d z .
\end{gathered}
$$

All these integrals from $I_{1}$ to $I_{12}$ are positive definite.

Now, putting $\sigma=i \sigma_{i}$ (where $\sigma_{i}$ is real) in Eq. (23) and equating the imaginary part, we get,

$$
\sigma_{i}\left\{\frac{1}{\varepsilon} I_{1}-\frac{d^{2}}{\varepsilon} I_{4}+\frac{\mu_{e} \varepsilon \eta d^{2}}{4 \pi \rho_{0} v} p_{2} I_{8}-\frac{\mu_{e} \varepsilon \eta}{4 \pi \rho_{0} v} p_{2} I_{10}+\left(\frac{\lambda \alpha a^{2} \kappa_{T}}{\beta v}\right)\left(g_{0}-\frac{\gamma M_{0} \nabla H}{\rho_{0} \alpha \lambda}\right) E p_{1} I_{12}\right\}=0 .
$$

In the absence of magnetic field (hence hall current), Eq. (24) becomes,

$$
\sigma_{i}\left\{\frac{1}{\varepsilon} I_{1}+\frac{\mu_{e} \varepsilon \eta d^{2}}{4 \pi \rho_{0} v} p_{2} I_{8}+\left(\frac{\lambda \alpha a^{2} \kappa_{T}}{\beta v}\right)\left(g_{0}-\frac{\gamma M_{0} \nabla H}{\rho_{0} \alpha \lambda}\right) E p_{1} I_{12}\right\}=0 .
$$

If $\lambda g_{0} \geq \frac{\gamma M_{0} \nabla H}{\rho_{0} \alpha}$, then the terms in bracket are positive definite, which implies that $\sigma_{i}=0$. Therefore, oscillatory modes are not allowed and the principle of exchange of stabilities is satisfied if $\lambda g_{0} \geq \frac{\gamma M_{0} \nabla H}{\rho_{0} \alpha}$.

\section{Numerical Discussion}

The dispersion relation (20) is analyzed numerically also. The numerical value of thermal Rayleigh number $R_{1}$ is decided for numerous values of medium permeability parameter $P$, couple-stress parameter $F_{1}$, magnetic field parameter $Q_{1}$, Hall current parameter $M_{h}$ and magnetization parameter $M_{0}$. Also, graphs have been plotted between $R_{1}$ and these parameters as shown in Figures (2) - (15),

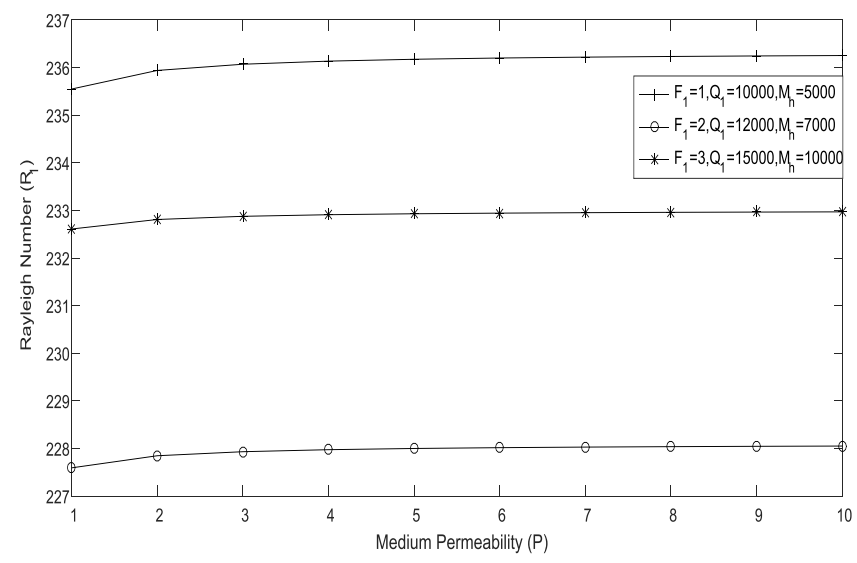

Fig. 2. Variation of $R_{1}$ with $P$ for $\lambda>0$. 


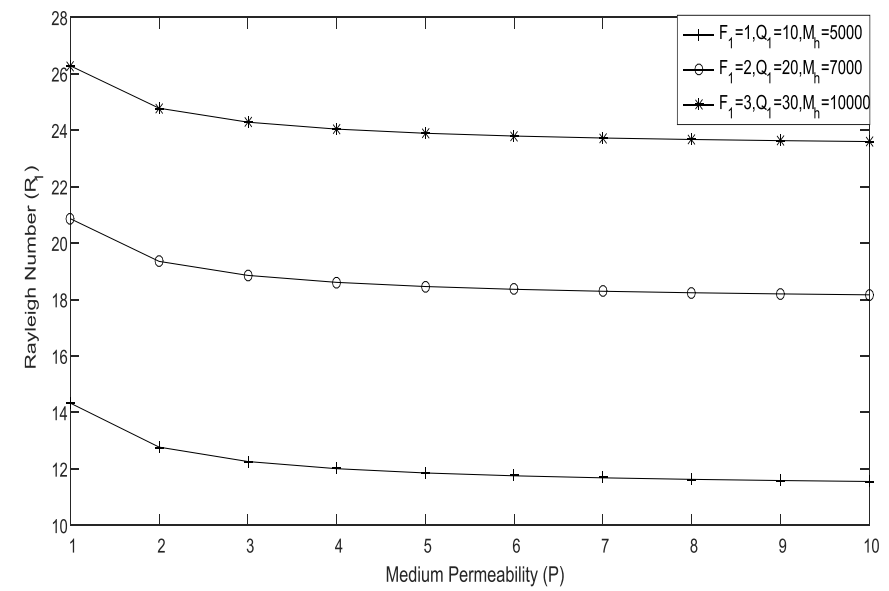

Fig. 3. Variation of $R_{1}$ with $P$ for $\lambda>0$.

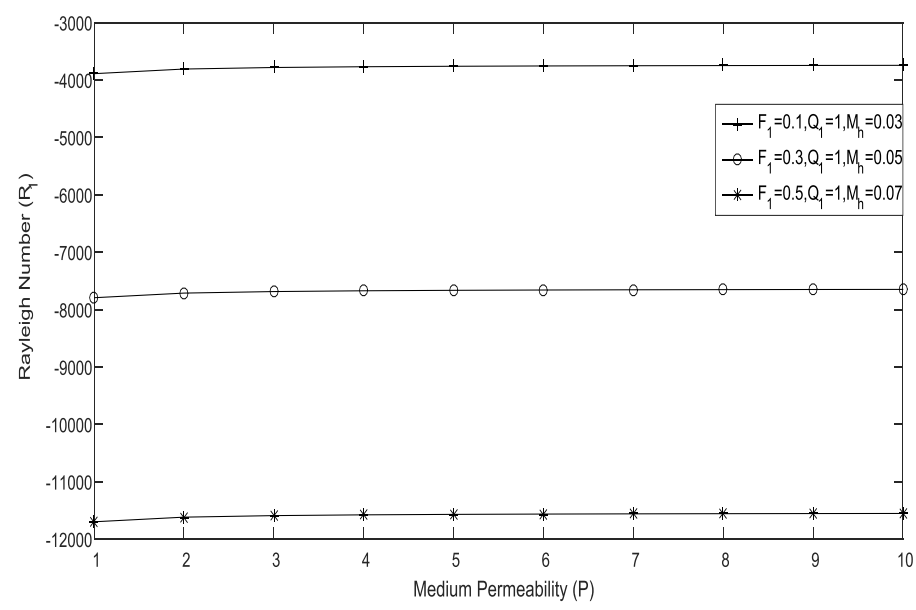

Fig. 4. Variation of $R_{1}$ with $P$ for $\lambda<0$.

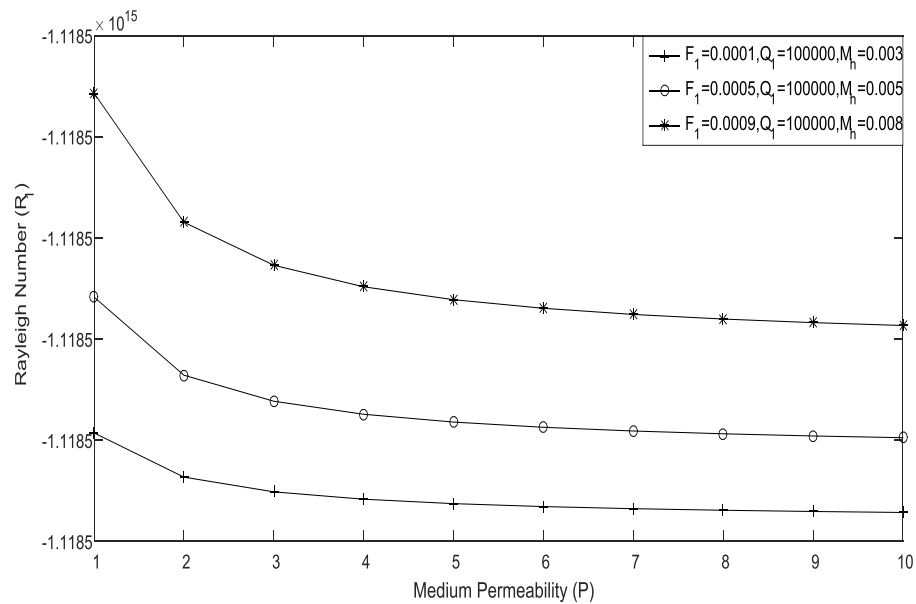


Fig. 5. Variation of $R_{1}$ with $P$ for $\lambda<0$.

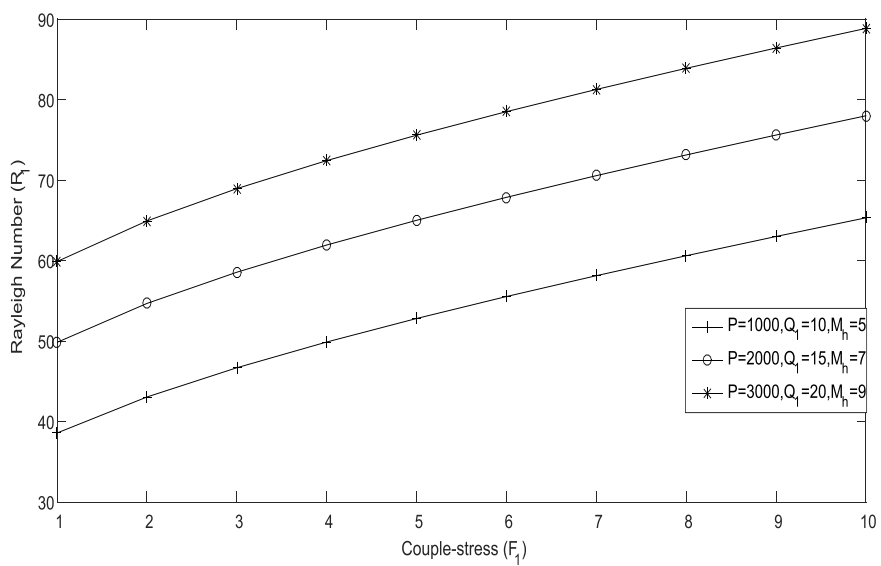

Fig. 6. Variation of $R_{1}$ with $F_{1}$ for $\lambda>0$.

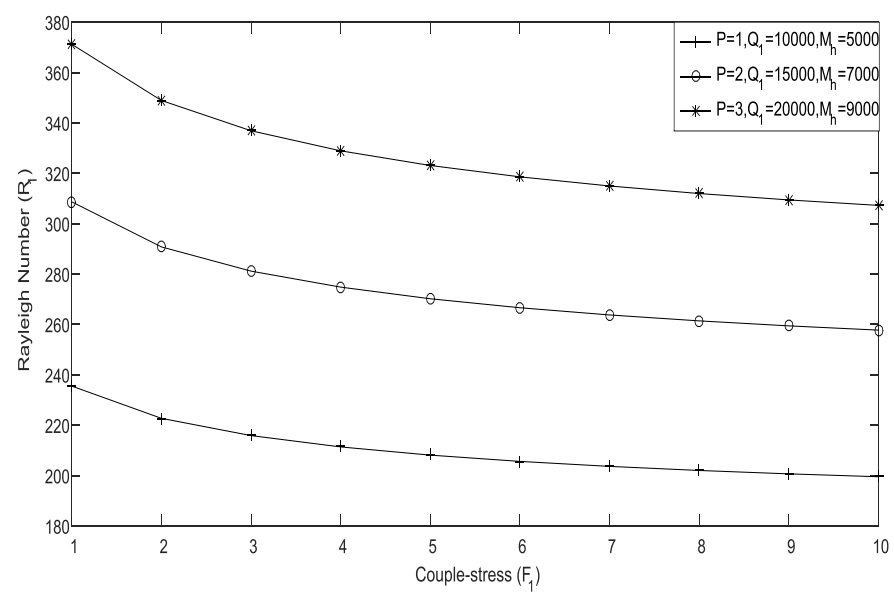

Fig. 7. Variation of $R_{1}$ with $F_{1}$ for $\lambda>0$.

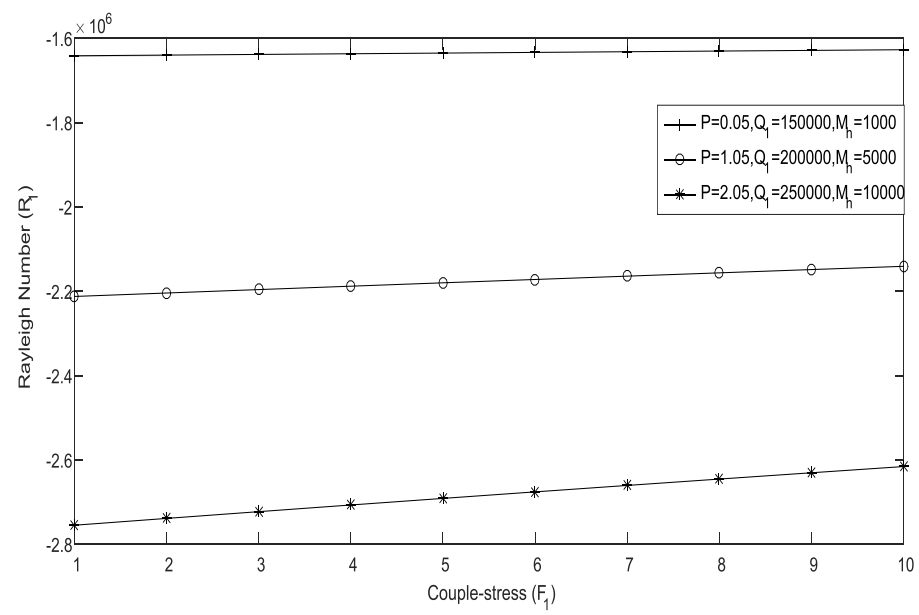


Fig. 8. Variation of $R_{1}$ with $F_{1}$ for $\lambda<0$.

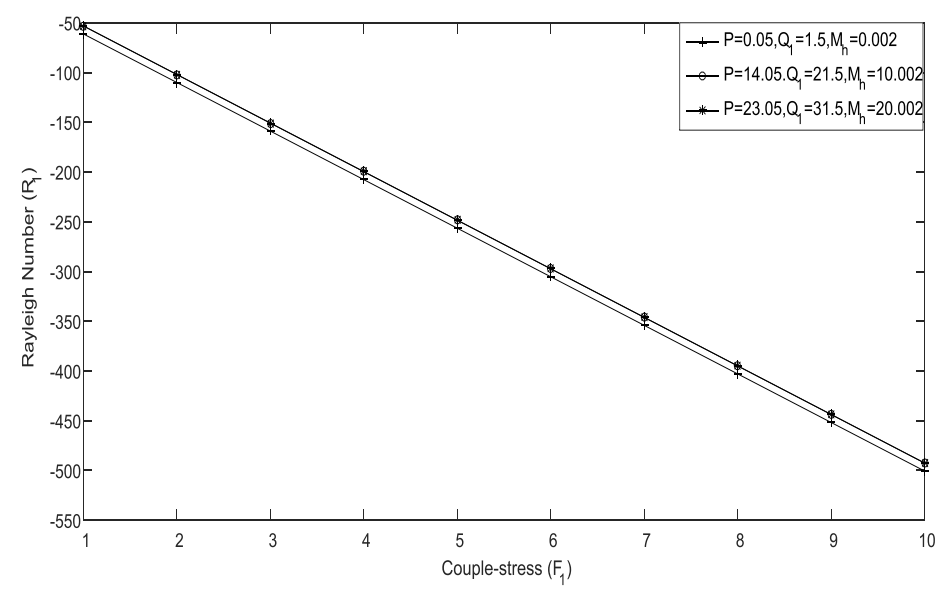

Fig. 9. Variation of $R_{1}$ with $F_{1}$ for $\lambda<0$.

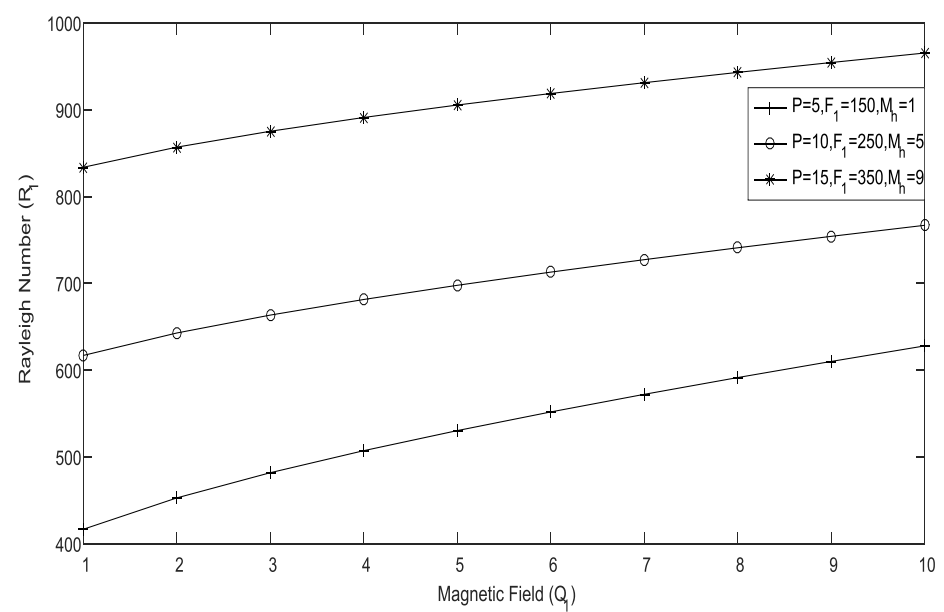

Fig. 10. Variation of $R_{1}$ with $Q_{1}$ for $\lambda>0$.

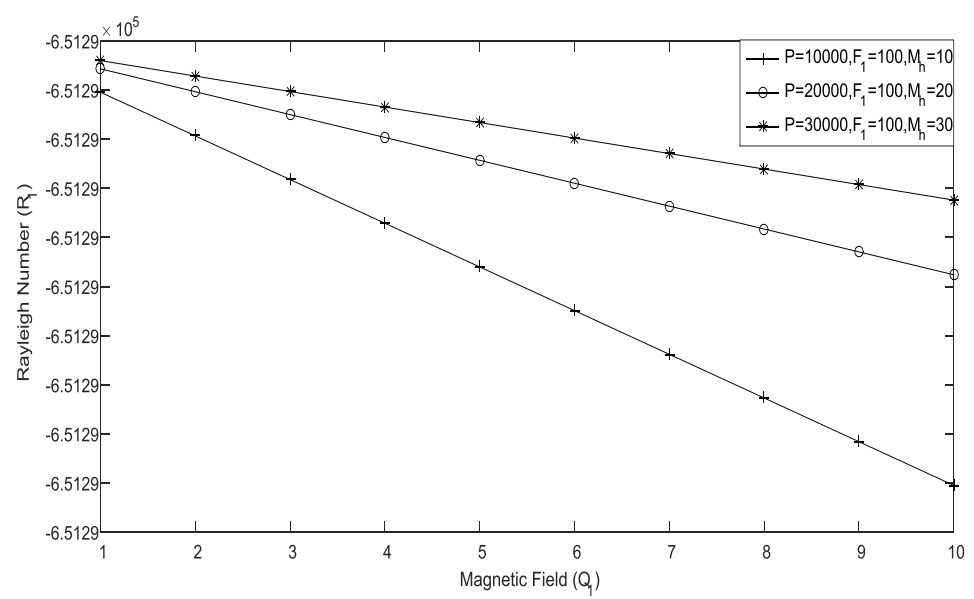


Fig. 11. Variation of $R_{1}$ with $Q_{1}$ for $\lambda<0$.

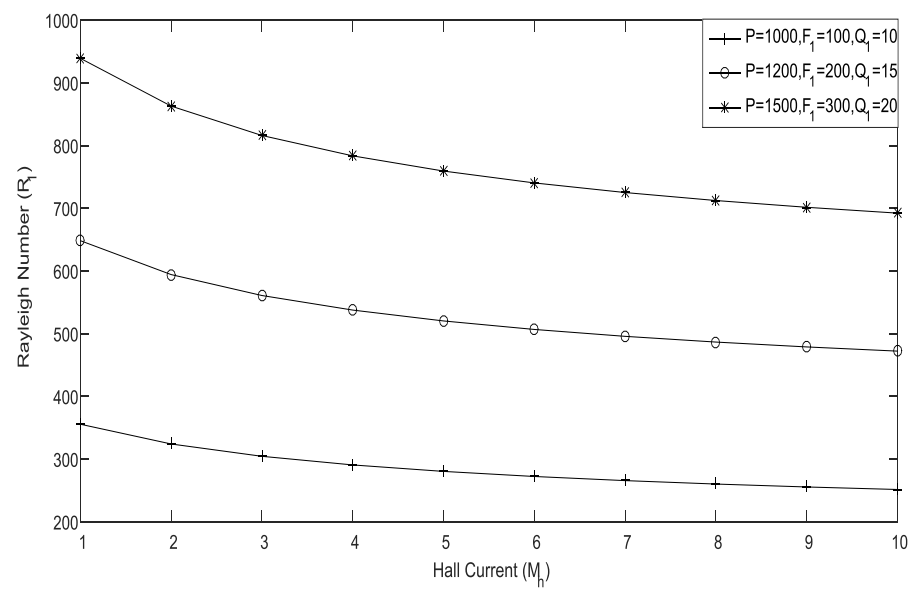

Fig. 12. Variation of $R_{1}$ with $M_{h}$ for $\lambda>0$.

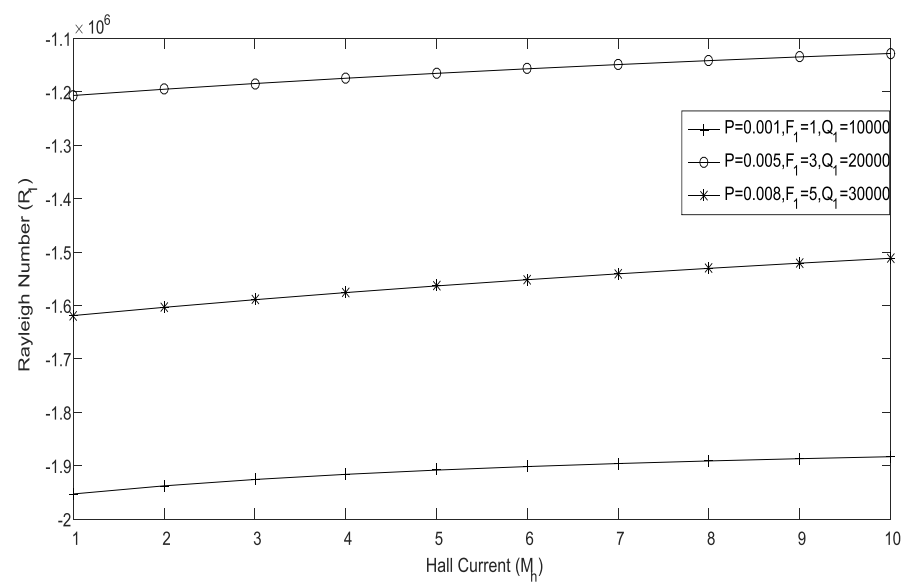

Fig. 13. Variation of $R_{1}$ with $M_{h}$ for $\lambda<0$.

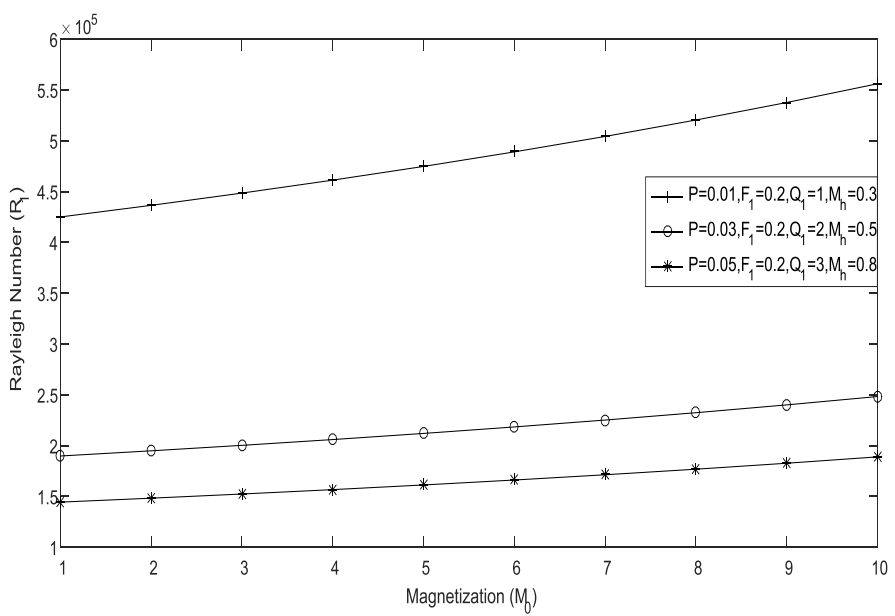


Fig. 14. Variation of $R_{1}$ with $M_{0}$ for $\lambda>0$.

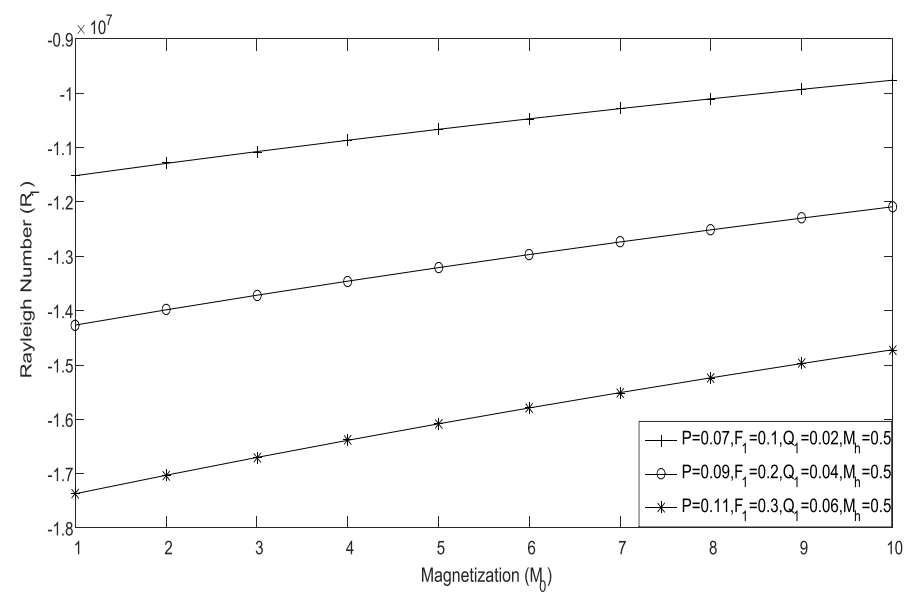

Fig. 15. Variation of $R_{1}$ with $M_{0}$ for $\lambda<0$.

In fig. 2, critical Rayleigh number $R_{1}$ increases with increase in medium permeability parameter $P$ for $\lambda=50$, which indicates that medium permeability has a stabilizing effect on the system. In fig. 3, critical Rayleigh number $R_{1}$ decreases with increase in medium permeability parameter $P$ for $\lambda=2$, which indicates that medium permeability has a destabilizing effect on the system. In fig. 4, critical Rayleigh number $R_{1}$ increases with increase in medium permeability parameter $P$ for $\lambda=-5$, which indicates that medium permeability has a stabilizing effect on the system. In fig. 5 , critical Rayleigh number $R_{1}$ decreases with increase in medium permeability parameter $P$ for $\lambda=-0.00001$, which indicates that medium permeability has a destabilizing effect on the system. In fig. 6, critical Rayleigh number $R_{1}$ increases with increase in couple-stress parameter $F_{1}$ for $\lambda=5$, which indicates that couple-stress has a stabilizing effect on the system. In fig. 7, critical Rayleigh number $R_{1}$ decreases with increase in couple-stress parameter $F_{1}$ for $\lambda=50$, which indicates that couple-stress has a destabilizing effect on the system. In fig. 8, critical Rayleigh number $R_{1}$ increases with increase in couple-stress parameter $F_{1}$ for $\lambda=-10000$, which indicates that couple-stress has a stabilizing effect on the system. In fig. 9, critical Rayleigh number $R_{1}$ decreases with increase in couple-stress parameter $F_{1}$ for $\lambda=-2000$, which indicates that couplestress has a destabilizing effect on the system.

In fig. 10, critical Rayleigh number $R_{1}$ increases with increase in magnetic field parameter $Q_{1}$ for $\lambda=3$, which indicates that magnetic field has a stabilizing effect on the system. In fig. 11, critical Rayleigh number $R_{1}$ decreases with increase in magnetic field parameter $Q_{1}$ for $\lambda=-15$, which indicates that magnetic field has a destabilizing effect on the system.

In fig. 12, critical Rayleigh number $R_{1}$ decreases with increase in hall current parameter $M_{h}$ for $\lambda=4$, which indicates that magnetic field has a destabilizing effect on the system. In fig. 13, 
critical Rayleigh number $R_{1}$ increases with increase in hall current parameter $M_{h}$ for $\lambda=-0.5$, which indicates that magnetic field has a stabilizing effect on the system.

In fig. 14, critical Rayleigh number $R_{1}$ increases with increase in magnetization parameter $M_{0}$ for $\lambda=0.2$, which indicates that magnetic field has a stabilizing effect on the system. In fig. 15, critical Rayleigh number $R_{1}$ increases with increase in magnetization parameter $M_{0}$ for $\lambda=-0.25$, which indicates that magnetic field has a stabilizing effect on the system.

\section{Conclusions}

In the present paper, we are discussing about the effect of hall current on thermal stability of couple-stress ferromagnetic fluid in the presence of variable gravity field and horizontal magnetic field saturating in a porous medium. A linearized theory and normal mode technique are used to attain the dispersion relation. The main results from the evaluation of the present paper are as below:

1. Medium permeability has both stabilizing and destabilizing effect on the system for $\lambda>0$ and $\lambda<0$ under certain conditions. Furthermore, in the absence of magnetic field, medium permeability has a stabilizing effect on the system for $\lambda<0$ and destabilizing effect for $\lambda>0$.

2. Couple-stress has both stabilizing and destabilizing effect on the system for $\lambda>0$ and $\lambda<0$ under certain conditions. Furthermore, in the absence of magnetic field, couplestress has a stabilizing effect on the system for $\lambda>0$ and destabilizing effect for $\lambda<0$.

3. Magnetic field has a stabilizing effect on the system for $\lambda>0$ and destabilizing effect for $\lambda<0$.

4. Hall current has a stabilizing effect on the system for $\lambda<0$ and destabilizing effect for $\lambda>0$.

5. Magnetization has a stabilizing effect on the system for both $\lambda>0$ and $\lambda<0$.

6. The principle of exchange of stabilities is not valid for the present problem under consideration, whereas in the absence of magnetic field (hence hall current), it is valid for the present problem if $\lambda g_{0} \geq \frac{\gamma M_{0} \nabla H}{\rho_{0} \alpha}$.

\section{Acknowledgment}

The corresponding author acknowledges CSIR, New Delhi for providing financial assistance through JRF Vide letter No. 08/688(0001)/2018-EMR-I.

\section{References}

[1] V.K. Stokes, “Couple-stresses in fluid,” Physics of Liquids, vol. 9, 1966, pp. 1709-1715.

[2] V.K. Stokes, "Theories of liquids with microstructure," Springer-Verlag, New-York, 1984.

[3] Sunil, Y.D.Sharma, P.K.Bharti and R.C.Sharma, "Thermosolutal instability of compressible Rivlin-Ericksen fluid with hall currents," International Journal of Applied Mechanics and Engineering, vol. 10, issue 2, 2005, pp. 329-343.

[4] N. Rani and S.K.Tomar, "Thermal convection problem of micropolar fluid subjected to hall current," Applied Mathematical Modeling, vol. 34, 2010, pp.508-519. 
[5] N. Rani and S.K. Tomar, "Double diffusive convection of micropolar fluid with hall currents," Int. J. of Applied Math. and Mech., vol. 6, issue 19, 2010, pp. 67-85.

[6] P. Kumar, "Effect of hall currents on thermal instability of compressible dusty viscoelastic fluid saturated in a porous medium," Studia Geotechnica et Mechnica, vol. XXXIII, issue 4, 2011, pp. 25-38.

[7] S. Chandrasekhar, "Hydrodynamic and Hydromagnetic Stability," Dover Publication, New York, 1981.

[8] R.E. Rosensweig, "Ferrohydrodynamics," Cambridge University Press, Cambridge, UK, 1985.

[9] B.A. Finlayson, "Convective instability of ferromagnetic liquids," Journal of Fluid Mechanics, vol. 40, issue 4, 1970, pp. 753-767.

[10] M.R. Raghavachar and V.S. Gothandaraman, "Hydromagnetic convection in a rotating fluid layer in the presence of hall current," Geophys. Astro. Fluid Dyn., vol. 45, issue 3-4, 1988, pp. 199-211.

[11] R.C. Sharma, Sunil and S. Chand,"Hall effect on thermal instability of Rivlin-Ericksen fluid," Indian J. Pure Appl. Math., vol. 31, issue 1, 2000, pp. 49-59.

[12] U. Gupta and P. Aggarwal,"Thermal instability of compressible Walters' (Model B') fluid in the presence of hall currents and suspended particles," Thermal Science, vol. 15, issue 2, 2011, pp. 487-500.

[13] U. Gupta, P. Aggarwal and R.K. Wanchoo,"Thermal convection of dusty compressible Rivlin-Ericksen fluid with hall currents," Thermal Science, vol. 16, issue 1, 2012, pp. 177191.

[14] A.K. Aggarwal and S. Makhija, "Hall effect on thermal stability of ferromagnetic fluid in porous medium in the presence of horizontal magnetic field," Thermal Science, vol. 18, Suppl. 2, 2014, pp. S503-S514.

[15] P.K. Nadian, R. Pundir and S.K. Pundir, "Thermal instability of rotating couple-stress ferromagnetic fluid in the presence of variable gravity field," Journal of Critical Reviews, vol. 7, issue 19, 2020, pp. 1842-1856.

[16] P.K. Nadian, R. Pundir and S.K. Pundir, "Thermal instability of couple-stress ferromagnetic fluid in the presence of variable gravity field, rotation and magnetic field" Journal of Critical Reviews, vol. 7, issue 19, 2020, pp. 2784-2797.

[17] P.K. Nadian, R. Pundir and S.K. Pundir, "Effect of rotation on couple-stress ferromagnetic fluid heated and soluted from below in the presence of variable gravity field," Journal of Critical Reviews, vol. 7, issue 10, 2020, pp. 2976-2986.

[18] S.K. Pundir, P.K. Nadian and R. Pundir, "Effect of dust particles on couple-stress ferromagnetic fluid heated from below in the presence of rotation and horizontal magnetic 
field saturating in a porous medium," Compliance Engineering Journal, vol. 11, issue 10, 2020, pp. 1-14. 